\title{
EXTENSIONS OF HOMOMORPHISMS
}

\section{PAUl Crvin}

1. Introduction. A multiplication was introduced by R. Arens [1] [2] into the second conjugate space $B^{* *}$ of a Banach algebra, $B$, which made $B^{* *}$ into a Banach algebra. The algebra of the second conjugate space was studied by Civin and Yood [3], with particular attention given to the case where $B$ was $L(\mathbb{S})$, the group algebra of the locally compact abelian group (5. Among the results they noted was that the algebra $M(\mathbb{S})$ of finite regular Borel measures on $(5)$ was isomorphic as an algebra with a quotient algebra of $L^{* *}(\mathbb{S})$. With $\mathfrak{S}$ also a locally compact abelian group, P. J. Cohen showed [4, p. 220] that any homomorphism of $L(\mathbb{S})$ into $M(\mathfrak{S})$ has an extension which was a homomorphism of $M(\mathbb{S})$ into $M(\mathfrak{E})$.

In $\S 3$ we discuss the extensions of homomorphisms defined on a Banach algebra $A$ into either the second conjugate algebra $B^{* *}$ of a Banach algebra $B$ or certain of its quotient algebras. The result of Cohen quoted above is included in Theorem 3.7 when $B S$ and $\mathfrak{S}$ are compact groups. In $\S 4$ we indicate, for compact $\mathfrak{S}$, a class of homomorphisms from $L(\mathfrak{S})$ into $M(\mathfrak{S})$, which are induced by homomorphisms of $L(\mathbb{S})$ into $L^{* *}(\mathfrak{S})$.

2. Notation. The notation of Civin and Yood [3] is used throughout. If $A$ is a Banach algebra, $A^{*}, A^{* *}, \cdots$ denote the various conjugate spaces of $A$. For $f \in A^{*}, x \in A,\langle f, x\rangle \in A^{*}$ is defined by $\langle f, x\rangle(y)=$ $f(x y), y \in A$. For $F \in A^{* *}, f \in A^{*},[F, f] \in A^{*}$ is defined by $[F, f](x)=$ $F(\langle f, x\rangle), x \in A$. Also for $F \in A^{* *}, G \in A^{* *}$ the multiplication $F G$ is defined in $A^{* *}$ by $F G(f)=F([G, f]), f \in A^{*}$.

For some purposes, Arens [2] also considers a second multiplication $F \cdot G$ defined for $F$ and $G$ in $A^{* *}$ in a manner similar to the above, except that at the first stage, $\langle f \mid x\rangle \in A^{*}$ is defined by $\langle f \mid x\rangle(y)=f(y x)$, $f \in A^{*}, x, y \in A$. Arens calls the multiplication in $A$ regular provided that $F \cdot G=G F$ for all $F, G \in A^{* *}$. Clearly, if $A$ is commutative, then $A^{* *}$ is commutative if and only if the multiplication in $A$ is regular. The same notation as above, in terms of bilinear functionals, is used in the sequel with respect to a multiplication in $A^{* * * *}$ which comes from the first of the above multiplications in $A^{* *}$.

If $\pi$ is the natural mapping of $A$ into $A^{* *}$, we say that a mapping $\varphi$ defined on $A^{* *}$ into a set $\subseteq$ is an extension of a mapping $\rho$ defined on $A$ into $\subseteq$ if $\varphi(\pi x)=\rho(x)$ for $x \in A$.

For any subset $\mathfrak{\Im}$ in $A^{*}$, we use the notation $\Im^{\perp}$ for $\left\{F \in A^{* *} \mid F(f)=\right.$ $0, f \in \Im\}$.

Received December 12, 1960. This research was supported by the National Science Foundation, grant NSF-G-14, 111. 
For a commutative Banach algebra $A$, we let $\mathfrak{Y}(A)$ denote the closed subspace of $A^{*}$ generated by the multiplicative linear functionals. If $A=L(\$)$, the group algebra of the locally compact group (\$), we write $\mathfrak{Y}(\mathbb{S})$ in place of $\mathfrak{Y}(L(\mathbb{S}))$.

3. Extension of homomorphisms. We first consider the possibility of extending a bounded homomorphism of the Banach algebra $A$ into the Banach algebra $B^{* *}$ to a $w^{*}$-continuous homomorphism of $A^{* *}$ into $B^{* *}$. Throughout this section we adopt the notation $\pi$ for the natural mapping of $A$ into $A^{* *}$ and $\sigma$ for the natural mapping of $B^{*}$ into $B^{* * *}$.

3.1 TheOREM. Let $A$ and $B$ be Banach algebras. Let $\phi$ be a bounded homomorphism of $A$ into the center of $B^{* *}$. Then there is a unique $w^{*}$-continuous homomorphism $\psi$ of $A^{* *}$ into $B^{* *}$ which is the extension of $\phi$.

Proof. Let $f \in B^{* *}$, and $x, y \in A$. Then $\left\langle\phi^{*} \sigma f, x\right\rangle(y)=\phi^{*} \sigma f(x y)=$ $\varphi(x y)(f)=\varphi(y) \varphi(x)(f)=\varphi(y)([\varphi(x), f])=\phi^{*} \sigma[\varphi(x), f](y) . \quad$ Thus $\left\langle\varphi^{*} \sigma f, x\right\rangle=\varphi^{*} \sigma[\varphi(x), f]$. For any $G \in A^{* *},\left[G, \varphi^{*} \sigma f\right](x) G\left(\left\langle\varphi^{*} \sigma f, x\right\rangle\right)=$ $G\left(\varphi^{*} \sigma[\varphi(x), f]=\sigma^{*} \phi^{* *} G([\varphi(x), f])=\sigma^{*} \varphi^{* *} G \varphi(x)(f)=\phi(x) \sigma^{*} \phi^{* *} G(f)=\right.$ $\varphi(x)\left(\left[\sigma^{*} \phi^{* *} G, f\right]\right)=\varphi^{*} \sigma\left[\sigma^{*} \varphi^{* *} G, f\right](x)$. Consequently, $\left[G, \varphi^{*} \sigma f\right]=$ $\phi^{*} \sigma\left[\sigma^{*} \phi^{* *} G, f\right]$. Therefore for any $F \in A^{* *}, F\left(\left[G, \varphi^{*} \sigma f\right]\right)=$ $F\left(\phi^{*} \sigma\left[\sigma^{*} \varphi^{* *} G, f\right]\right)=\sigma^{*} \varphi^{* *} F\left(\left[\sigma^{*} \varphi^{* *} G, f\right]\right)$. Hence $\sigma^{*} \phi^{* *}(F G)(f)=$ $F G\left(\phi^{*} \sigma f\right)=F\left(\left[G, \varphi^{*} \sigma f\right]\right)=\sigma^{*} \phi^{* *} F\left(\left[\sigma^{*} \varphi^{* *} G, f\right]\right)=\sigma^{*} \phi^{* *} F \sigma^{*} \phi^{* *} G(f)$. Thus $\sigma^{*} \varphi^{* *}$ is a homomorphism of $A^{* *}$ into $B^{* *}$.

For $x \in A$, and $f \in B^{*}, \sigma^{*} \phi^{* *}(\pi x)(f)=\pi x\left(\phi^{*} \sigma f\right)=\varphi^{*} \sigma f(x)=\sigma f(\varphi(x))=$ $\varphi(x)(f)$. Thus $\sigma^{*} \phi^{* *}(\pi x)=\varphi(x)$ and $\sigma^{*} \varphi^{* *}$ is an extension of $\varphi$.

Let $G \in A^{* *}, G_{\alpha} \in A^{* *}$ and suppose $G=w^{*}-\lim G_{\alpha}$. Then for any $f \in B^{*}, \lim \sigma^{*} \varphi^{* *} G_{\alpha}(f)=\lim G_{\alpha}\left(\varphi^{*} \sigma f\right)=\sigma^{*} \varphi^{* *} G(f)$, and so $\sigma^{*} \varphi^{* *}$ is $w^{*}$-continuous.

The assertion of uniqueness follows from the following.

3.2 Lemma. Let $A$ and $B$ be Banach algebras, and let $\phi$ be any bounded linear transformation of $A$ into $B^{* *}$. Then $\sigma^{*} \phi^{* *}$ is the only $w^{*}$-continuous extension of $\varphi$ to a transformation of $A^{* *}$ into $B^{* *}$.

Proof. That $\sigma^{*} \phi^{* *}$ is a $w$-continuous extension was given above. Suppose that $\psi$ is a $w^{*}$-continuous extension of $\varphi$, so that $\psi(\pi x)=\varphi(x)$ for all $x \in A$. Let $G \in A^{* *}$ and let $\left\{x_{\alpha}\right\}$ be a net in $A$ such that $w^{*}$-lim $\pi x_{\alpha}=G$. Then for $f \in B^{*}, \psi(G)(f)=\lim \psi\left(\pi x_{\alpha}\right) f=\lim \varphi\left(x_{\alpha}\right)(f)=\lim$ $\varphi^{*} \sigma f\left(x_{\alpha}\right)=\lim \pi x_{\alpha}\left(\varphi^{*} \sigma f\right)=G\left(\phi^{*} \sigma f\right)=\sigma^{*} \varphi^{* *} G(f)$. Hence $\psi(G)=$ $\sigma^{*} \phi^{* *} G$.

If $B$ is commutative with a regular multiplication, an alternative proof of Theorem 3.1 may be given on the basis of the following lemma and Theorem 6.1 of [3]. 
3.3 Lemma. If $B$ is a commutative Banach algebra with a regular multiplication then $\sigma^{*}$ is a homomorphism of $B^{* * * *}$ into $B^{* *}$.

Proof. Since multiplication in $B$ is regular, $B^{* *}$ is [2] a commutative algebra. Let $U, V \in B^{* * * *}$. For $f \in B^{*}$, and $F, G \in B^{* *},\langle\sigma f, F\rangle(G)=$ $\sigma f(F G)=F G(f)=G F(f)=G([F, f])=\sigma[F, f](G)$, and therefore $\langle\sigma f, F\rangle=\sigma[F, f]$. Also $[V, \sigma f](F)=V(\langle\sigma f, F\rangle)=V(\sigma[F, f])=$ $\sigma^{*} V[F, f]=\left(\sigma^{*} V\right) F(f)=F \sigma^{*} V(f)=F\left(\left[\sigma^{*} V, f\right]\right)=\sigma\left[\sigma^{*} V, f\right](F)$. Thus $[V, \sigma f]=\sigma\left[\sigma^{*} V, f\right]$. Consequently $\sigma^{*}(U V)(f)=U V(\sigma f)=U([V, \sigma f])=$ $U\left(\sigma\left[\sigma^{*} V, f\right]\right)=\sigma^{*} U\left(\left[\sigma^{*} V, f\right]\right)=\sigma^{*} U \sigma^{*} V(f)$ and $\sigma^{*}$ is a homomorphism a claimed.

We note that it is impossible in general to conclude that the range of the extension of $\varphi$ is in the center of $B^{* *}$ even though the range of $\phi$ is in the center. For let $A=B$ be a commutative algebra whose multiplication is not regular, and let $\varphi=\pi$. Then the $w^{*}$-continuous extension of $\pi$ is the identity map and $B^{* *}$ is not commutative.

One further example is in order, to see that in general a bounded homomorphim $\varphi$ from $A$ into $B^{* *}$ does not admit a $w^{*}$-continuous extension as a homomorphism from $A^{* *}$ into $B^{* *}$. For this purpose let $A$ be the group algebra of the integers, (S), and let $B=A$. Let $t_{\gamma}, \gamma \in \mathbb{B}$ be the translation operator on $A^{*}$, defined by $t_{\gamma} f(\alpha)=f(\alpha+\gamma), f \in A^{*}$, and $\alpha$, $\gamma \in$ (S). Let $e \in A^{*}$ correspond to the function identically one on $\mathbb{S}$. Let $\mathfrak{\Im}=\left\{F \in A^{* *} \mid F\left(t_{\gamma} f\right)=F(f)\right.$, for all $\left.\gamma \in \mathbb{B}, f \in A^{*}\right\}$. Then as noted in formula (3.2) of [3],

$$
G F=G(e) F, F \in \Im, G \in A^{* *} .
$$

In particular any $F \in \mathfrak{\Im}$ with $F(e)=1$ is an idempotent. As noted in [3], $\mathfrak{\Im}$ is a two sided ideal in $A^{* *}$ with only zero in common with the center of $A^{* *}$. Since $(S)$ is a discrete group $A$ has an identity and thus [3, Lemma 5.4] $A^{* *}$ has an identity $E$. Let $F$ be a nonzero idempotent in $\Im$. Thus $E-F$ is also an idempotent. Let $\varphi(x)=\pi x(E-F)$. Since $\pi A$ is in the center of $A^{* *}, \varphi(x)$ is a homomorphism of $A$ into $A^{* *}$. If $\varphi$ had a $w^{*}$-continuous extension as a homomorphism, the extension $\psi$ would have the value $\psi(G)=G(E-F), G \in A^{* *}$. We now show that $\psi$ is not a homomorphism. As noted above $F$ is not in the center of $A^{* *}$, so we may pick $H \in A^{* *}$ such that $H F \neq F H$. Also pick $G \in A^{* *}$ such that $G(e)=1$. Then $\psi(G H)=G H(E-F)=G H-G H F=$ $G H-(G H)(e) F$. Now $e$ is a multiplicative linear functional on $A$, and so by Lemma 3.6 of [3], $(G H)(e)=G(e) H(e)=H(e)$. Thus $\psi(G H)=$ $G H-H(e) F=G H-H F$. On the other hand $\psi(G) \psi(H)=(G-G F)(H-$ $H F)=(G-F)(H-H(e) F)=G H-F H-H(e) G F+H(e) F=G H-F H$. Since $F H \neq H F, \psi(G H) \neq \psi(G) \psi(H)$ and $\psi$ is not a homomorphism.

Before turning to other types of extensions we note one further 
item on the matter of $w^{*}$-continuity of homomorphisms.

3.4 Lemma. If $A$ and $B$ are Banach algebras and $\psi$ is a bounded homomorphism of $A^{* *}$ into the center of $B^{* *}$, then there is a $w^{*}$-continuous homomorphism $\rho$ of $A^{* *}$ into $B^{* *}$ such that $\psi(\pi x)=\rho(\pi x)$ for $x \in A$.

Proof. Since $\psi \pi$ is a homomorphism of $A$ into the center of $B^{* *}$, we may take $\rho=\sigma^{*} \psi^{* *} \pi^{* *}$ and apply Theorem 3.1.

Homomorphisms of $A^{* *}$ into $B^{* *}$ which are not $w^{*}$-continuous exist, as may be seen in the following example. Let $\mathbb{B}$ be an infinite compact group and let $A=B$ be the group algebra of $(5$. Then by Lemma 3.8 of [3], $A^{* *}$ has a right identity $E$ which is not an identity. Define for $F \in A^{* *}, \psi(F)=E F$. Then $\psi(F G)=E F G=E F E G=\psi(F) \psi(G)$. However $\psi$ although bounded is not $w^{*}$-continuous. For let $G \in A^{* *}$ and let $\left\{x_{\alpha}\right\}$ be a net such that $w^{*}-\lim \pi x_{\alpha}=G$. Then if $\psi$ were $w^{*}$-continuous we would have $\psi(G)=\lim \psi\left(\pi x_{\alpha}\right)=\lim E \pi x_{\alpha}=\lim \pi x_{\alpha}=$ $G$. However, $\psi(G)=E G$ and $E G \neq G$ for some $G \in A^{* *}$.

We next turn to the question of extending homomorphisms from $A$ into certain quotient algebras of $B^{* *}$ in the case in which both $A$ and $B$ are commutative. We must first characterize the $w^{*}$-closed ideals of a second conjugate algebra.

3.5 Lemma. Let $A$ be a commutative Banach algebra. Let $\Im$ be a $w^{*}$-closed subspace of $A^{* *}$ and let $\mathfrak{I}_{0}=\left\{f \in A^{*} \mid F(f)=0, F \in \Im\right.$ $\}$. Then $\mathfrak{\Im}$ is an ideal of $A^{* *}$ if and only if $[G, f] \in \mathfrak{\Im}_{0}$ for all $G \in A^{* *}, f \in \Im_{0}$.

Proof. Since $\mathfrak{\Im}$ is $w^{*}$-closed, $\mathfrak{\Im}=\mathfrak{Y}_{0}{ }^{\perp}$. Suppose $\mathfrak{\Im}$ is an ideal of $A^{* *}$. For any $F \in \mathfrak{\Im}, G \in A^{* *}$, and $f \in \mathfrak{\Im}_{0}, F G \in \Im$ and $F G(f)=0$. Therefore $F([G, f])=0$ for all $F \in \Im$, and so by definition $[G, f] \in \Im_{0}$. Suppose next that the stated condition holds. Let $F \in \mathfrak{J}$ and $G \in A^{* *}$. For any $f \in \mathfrak{\Im}_{0},[G, f] \in \mathfrak{\Im}_{0}$ and thus $F G(f)=F([G, f])=0$. Consequently $F G \in \mathfrak{\Im}_{0}^{\perp}=\mathfrak{\Im}$ and $\mathfrak{\Im}$ is a right ideal. For any $x \in A, \pi x$ is in the center of $A^{* *}$, hence if $F \in \mathfrak{\Im}, \pi x F=F \pi x \in \mathfrak{\Im}$. Since $\pi A$ is $w^{*}$-dense in $A^{* *}$ and left multiplication is $w^{*}$-continuous [2], we see that $G F \in \Im$ for any $G \in A^{* *}$, and thus $\mathfrak{\Im}$ is an ideal of $A^{* *}$.

3.6 Theorem. Let $A$ and $B$ be commutative Banach algebras. Let $\Im$ be a $w^{*}$-closed ideal of $B^{* *}$. Suppose that $\varphi$ is a bounded homomorphism of $A$ into the center of $B^{* *} / \Im$. Then there exists a $w^{*}$-closed ideal $\mathfrak{\Im}^{\prime}$ of $A^{* *}$ and a homomorphism $\psi$ of $A^{* *} / \Im^{\prime}$ into $B^{* *} / \Im$ such that if $\pi$ is the natural embedding of $A$ into $A^{* *}$, then $\psi\left(\pi x+\Im^{\prime}\right)=$ $\varphi(x), x \in A$. 
Proof. Since $\Im$ is $w^{*}$-closed, $\Im=\Im_{0}{ }^{\perp}$ where $\mathfrak{\Im}_{0}=\left\{f \in B^{*} \mid F(f)=0\right.$ for all $F \in \Im$ \}. Let $\beta$ be the linear space isometric isomorphism of $\Im_{0}^{*}$ onto $B^{* *} / \Im$ defined for $F_{0} \in \mathfrak{\Im}_{0}^{*}$ by $\beta F_{0}=F+\Im$ where $F \in B^{* *}$ is an arbitrary extension of $F_{0}$. Define multiplication in $\mathfrak{\Im}_{0}^{*}$ so that $\beta$ (and thus $\left.\beta^{-1}\right)$ is an algebra isomorphism. For $f \in \Im_{0}$, define $\varphi_{*} f$ by $\varphi_{*} f(x)=$ $\left(\beta^{-1} \varphi(x)\right)(f), x \in A$. Then $\mathcal{P}_{*} f$ is linear and since $\varphi$ is bounded $\left\|\varphi_{*} f(x)\right\| \leqq\|\varphi\|\|x\|\|f\|$, and $\varphi_{*} f \in A^{*}$.

Let $\Im_{0}^{\prime}$ be the $w^{*}$-closure of the range of $\varphi_{*}$, and let $\Im^{\prime}=\Im^{\prime} 0^{\perp}$. Clearly $\mathfrak{\Im}^{\prime}$ is $w^{*}$-closed. We next show that $\mathfrak{\Im}^{\prime}$ is an ideal of $A^{* *}$. Let $f \in \mathfrak{\Im}_{0}$. Then for any $x, y \in A,\left\langle\varphi_{*} f, x\right\rangle(y)=\varphi_{*} f(x y)=\left(\beta^{-1} \varphi(x y)\right) f=$ $\left(\beta^{-1} \varphi(y x)\right)(f)$, since the range of $\varphi$ is commutative. Suppose that $\phi(y)=$ $U+\Im$, and $\varphi(x)=V+\Im$ so that $\varphi(y x)=U V+\Im$. Then $\left(\beta^{-1} \varphi(x y)\right)(f)=$ $U V(f)=U([V, f])$. Since $f \in \mathfrak{\Im}_{0}$, and $\Im=\mathfrak{\Im}_{0}{ }^{\perp}$ is an ideal, $g=[V, f] \in \Im_{0}$ by Lemma 3.5. Hence $\left(\beta^{-1} \mathcal{Q}(y x)\right)(f)=U(g)=\left(\beta^{-1} \varphi(y)\right)(g)=\phi_{*} g(y)$, for all $y \in A$. We therefore have $\left\langle\varphi_{*} f, x\right\rangle=\mathscr{\varphi}_{*} g$ and so $\left\langle\varphi_{*} f, x\right\rangle \in \Im^{\prime}$ for any $x \in A$ and $f \in \widetilde{\Im}_{0}$. Suppose next that $g \in \widetilde{\Im}_{0}^{\prime}$, and $x \in A$. Say $g=$ $w^{*}-\lim \varphi_{*} f_{\alpha}$ with $f_{\alpha} \in \mathfrak{\Im}_{0}$. Then for $y \in A,\langle g, x\rangle(y)=g(x y)=\lim \varphi_{*} f_{\alpha}(x y)=$ $\lim \left\langle\varphi_{*} f_{\alpha}, \mathrm{x}\right\rangle(y)$, and hence $\langle g, x\rangle=w^{*}-\lim \left\langle\varphi_{*} f_{\alpha}, x\right\rangle$. However, by the above, $\left\langle\varphi_{*} f_{\alpha}, x\right\rangle \in \mathfrak{\Im}_{0}^{\prime}$, and $\Im_{0}^{\prime}$ is $w^{*}$-closed so $\langle g, x\rangle \in \mathfrak{\Im}_{0}^{\prime}$ for any $g \in \widetilde{\Im}_{0}^{\prime}$ and $x \in A$.

Let $G \in A^{* *}$ and let $f \in \mathfrak{\Im}_{0}{ }^{\prime}$. Let $\left\{x_{\alpha}\right\}$ be a net in $A$ such that $w^{*}-\lim \pi x_{\alpha}=G$. Then $[G, f](x)=G(\langle f, x\rangle)=\lim \pi x_{\alpha}(\langle f, x\rangle)=\lim$ $\langle f, x\rangle\left(x_{\alpha}\right)=\lim f\left(x x_{\alpha}\right)=\lim f\left\langle x_{\alpha} x\right)=\lim \left\langle f, x_{\alpha}\right\rangle(x)$ for $x \in A$. Consequently $[G, f]=w^{*}-\lim \left\langle f, x_{\alpha}\right\rangle$, and is thus in $\mathfrak{\Im}_{0}^{\prime}$ as $\mathfrak{\Im}_{0}^{\prime}$ is $w^{*}$-closed. Hence, by Lemma 3.5, $\mathfrak{\Im}^{\prime}=\mathfrak{\Im}_{0}^{\prime \perp}$ is a $w^{*}$ closed ideal of $\mathrm{A}^{* *}$.

For $F \in A^{* *}$, define $\gamma F(f)=F\left(\phi_{*} f\right)$ for $f \in \mathfrak{\Im}_{0}$. Clearly $\gamma F$ is a bounded linear functional on $\Im_{0}$, and so has an extension of the same norm which is an element of $B^{* *}$. We again denote the extension by $\gamma F$. Thus $\gamma$ is a bounded linear map from $A^{* *}$ into $B^{* *}$. Note that if $F_{1}-F_{2} \in \Im^{\prime}$ and $f \in \Im_{0}$, then $\gamma\left(F_{1}-F_{2}\right)(f)=\left(F_{1}-F_{2}\right)\left(\mathscr{P}_{*} f\right) 0$, and thus $\gamma F_{1}-\gamma F_{2} \in \mathfrak{\Im}$. Thus for any $F \in F_{0}+\mathfrak{J},\left\|\gamma F_{0}+\mathfrak{J}^{\prime}\right\|=\|\gamma F+\mathfrak{\Im}\| \leqq$ $\|\gamma F\| \leqq\|F\|\left\|\varphi_{*}\right\|$ and hence $\left\|\gamma F_{0}+\mathfrak{\Im}\right\| \leqq\left\|F_{0}+\mathfrak{\Im}^{\prime}\right\|\left\|\varphi_{*}\right\|$.

Define $\psi$ on $A^{* *} / \mathfrak{\Im}^{\prime}$ by $\psi\left(F+\mathfrak{\Im}^{\prime}\right)=\gamma F+\mathfrak{\Im}$. By the above, we see that $\psi$ is a bounded linear mapping of $A^{* *} / \mathfrak{F}^{\prime}$ into $B^{* *} / \Im$. Also for $x \in A, \psi\left(\pi x+\Im^{\prime}\right)=\gamma \pi x+\Im$. Since $\gamma \pi x(f)=\pi x\left(\varphi_{*} f\right)=\varphi_{*} f(x)=$ $\left(\beta^{-1} \varphi(x)\right)(f)$ for $f \in \mathfrak{I}_{0}, \gamma \pi x-\beta^{-1} \varphi(x) \in \mathfrak{I}$, and $\psi(\pi x+\mathfrak{J})^{\prime}=\varphi(x)$.

Thus all that remains is to see that $\psi$ satisfies the required multiplicative property of a homomorphism. Let $F, G \in A^{* *}$. To see that $\psi(F G)=\psi(F) \psi(G)$, we must show that for $f \in \Im_{0},\{\gamma(F) \gamma(G)-\gamma(F G)\}(f)=0$. Since $\{\gamma(F) \gamma(G)-\gamma(F G)\}(f)=\gamma(F)([\gamma(G), f])-F G\left(\mathscr{D}_{*} f\right)=F\left(\phi_{*}[\gamma(G), f]\right.$ $\left.\left[G, \Phi_{*} f\right]\right)$, it suffices if we show that $\varphi_{*}[\gamma(G), f]-\left[G, \Phi_{*} f\right]=0$. Let $x, y \in A$ and suppose that $\varphi(x)=U+\Im, \varphi(y)=V+\Im$, and thus $\varphi(x y)=$ $\phi(y x)=V U+\Im$. It follows that $\left\langle\varphi_{*} f, x\right\rangle(y)=\phi_{*} f(x y)=V U(f)=V([U, f])$. Now, since $f \in \Im_{0},[U, f] \in \Im_{0}$ by Lemma 3.5. We therefore have $\left\langle\Phi_{. .} f . x\right\rangle(y)=$ 
$\varphi_{*}[U, f](y)$ for all $y \in A$, and consequently $\left\langle\varphi_{*} f, x\right\rangle=\varphi_{*}[U, f]$. Thus $\left[G, \varphi_{*} f\right](x)=G\left(\left\langle\varphi_{*} f, x\right\rangle\right)=G\left(\varphi_{*}[U, f]\right)=\gamma G([U, f]=(\gamma G) U(f)$. On the other hand, $\varphi_{*}[\gamma G, f](x)=U([\gamma G, f])=U \gamma G(f)$. Since under our hypothesis $\varphi(x)=U+\mathfrak{\Im}$ is in the center of $B^{* *} / \mathfrak{\Im}, U \gamma G(f)=(\gamma G) U(f)$ for $f \in \mathfrak{J}_{0}$ and we have the desired result.

It should be noted that the ideal $\mathfrak{J}^{\prime}$ in general is dependent on the homomorphism $\varphi$. Two instances should be noted where this is not the case. The first, when $\mathfrak{\Im}^{\prime}=0$, has already been treated in the discussion of $w^{*}$-continuous extensions of homomorphisms of $A$ into the center of $B^{* *}$. The other is the following.

3.7 Theorem. Let $A$ and $B$ be commutative Banach algebras. Let $\varphi$ be a homomorphism of $A$ into $B^{* *} / \mathfrak{Y}^{\perp}(B)$. Then there is a homomorphism $\psi$ of $A^{* *} / \mathfrak{V}{ }^{\perp}(B)$ such that $\psi\left(\pi x+\mathfrak{Y}^{\perp}\right)=\varphi(x)$.

Proof. If in the proof of Theorem 3.6, $\mathfrak{\Im}_{0}=\mathfrak{Y}(B)$, it follows from Lemma 3.6 of [3] that for any $f \in \Im_{0}$ which is a multiplicative linear functional on $B$, that $\varphi_{*} f$ is a multiplicative linear functional on $A$. Hence, the norm closure of the range of $\varphi_{*}$ is contained in $\mathfrak{Y}(A)$. In view of Lemma 3.6 of [3], the subspace $\mathfrak{Y}^{\perp}(A)$ is a $w^{*}$-closed ideal of $A^{* *}$, and if used in the role of $\mathfrak{\Im}^{\prime}$ affords the same conclusion. Note that the homomorphism $\varphi$ is not postulated to be bounded or with range in the center of $B^{* *} / \mathfrak{Y}^{\perp}(B)$. This is legitimate since in view of Theorem 3.7 of [3], $B^{* *} / \mathfrak{Y}^{\perp}$ is automatically commutative and semi-simple, and thus $\varphi$ is automatically bounded.

If $A$ and $B$ are the group algebras of the compact groups $B$ and $\mathfrak{S}$, then $A^{* *} / \mathfrak{Y}{ }^{\perp}(A)$ and $B^{* *} / \mathfrak{I}{ }^{\perp}(B)$ may be identified with the measure algebras $M(\mathbb{S})$ and $M(\mathfrak{E})$ respectively by Theorem 3.18 of [3]. Thus Theorem 3.7 includes in the case of compact groups, the result of P. J. Cohen [4] quoted in the introduction.

4. Group algebras. Let $(S)$ be a locally compact abelian group. As in $\S 3$, we denote the group algebra of $\mathbb{B S}$ by $L(\mathbb{B})$ and the algebra of finite regular Borel measures on $\mathbb{B}$ by $M(\mathbb{S})$. For notational purposes, it is also convenient to identify the character group $\hat{B}$ of $\mathbb{B}$ with the subset of $L^{*}(\mathbb{S})$ consisting of the nonzero multiplicttive linear functional on $L(\mathbb{B})$. The topology of $\hat{\mathbb{S}}$ is then in agreement with the $w^{*}$-topology of $\hat{B}$ as a subset of $L^{*}(\mathbb{S})$.

Suppose that $\mathfrak{K}$ is a locally compact abelian group. A continuous homomorphism $\nu$ of $\mathbb{B}$ into $\mathfrak{S}$ is called nonsingular if for every Borel set $E$ is $\mathfrak{S}$ with zero Haar measure, $\nu^{-1}(\mathfrak{F})$ is of zero Haar measure in $\mathbb{B}$.

A complete characterization of all homomorphisms $\varphi$ of $L(\mathbb{S})$ into $M(\mathfrak{S})$ was given by P. J. Cohen [4]. He utilized the function $\varphi_{*}$ from $\hat{\mathfrak{S}}$ into $\{\hat{\mathscr{S}}, 0\}$ defined by $\varphi_{*} f(x)=\varphi(x)(f), x \in L(\mathbb{S}), f \in \hat{\mathfrak{S}}$. 
4.1 TheoRem. (P. J. Cohen) Let \&S and $\mathfrak{S}$ be tocally compact abelian groups, $\rho$ a homomorphism of $L(\mathbb{S})$ into $M(\mathfrak{\$}), \varphi_{*}$ the induced map of $\hat{\mathfrak{S}}$ into, $\{\hat{S}, 0\}$. Then there are a finite number of sets $\AA_{i}$, which are cosets of open subgroups of $\hat{\mathfrak{S}}$, and continuous maps $\psi_{i}: \hat{\mathfrak{\aleph}}_{i} \rightarrow \mathfrak{B}$, such that

$$
\psi_{i}(x+y-z)=\psi_{i}(x)+\psi_{i}(y)-\psi_{i}(z)
$$

for all $x, y$ and $z$ in $\AA_{i}$, with the following property: There is a decomposition of $\hat{\mathfrak{S}}$ into the disjoint union of sets $\mathfrak{S}_{j}$, each lying in the Boolean ring generated by the sets $\mathfrak{R}_{i}$, such that on each $\widetilde{S}_{j}, \Phi_{*}$ is either identically zero or agrees with some $\psi_{i}$, where $\widetilde{S}_{j} \subset \Re_{i}$.

Conversely, for any map of $\hat{\mathfrak{S}}$ into $\{\hat{\mathfrak{S}}, 0\}$, there is a homomorphism of $L(\mathbb{S})$ into $M(\mathfrak{S})$ which induces it. The map $\phi$ carries $L(\mathbb{S})$ into $L(\mathfrak{S})$ if and only if ${\Phi_{*}}^{-1}$ of every compact subset of $\hat{\text { SO }}$ is compact.

Suppse that the sets $\mathscr{R}_{i}$ are cosets of the subgroups $\mathfrak{U}_{i}$ of $\hat{\mathfrak{S}}$. There is a closed subgroup $\mathfrak{S}_{i}$ of $\mathfrak{S}_{2}, \mathfrak{S}_{i}=\left\{h \in \mathfrak{S} \mid(h, \hat{h})=1, \hat{h} \in \mathfrak{U}_{i}\right\}$, such that $\mathfrak{U}_{i}$ may be viewed [6, p. 130] as the character group of $\mathfrak{S}_{2} / \mathfrak{S}_{i}$. Let $a_{i} \in \mathfrak{R}_{i}$, and define $\psi_{i}^{\prime}: \mathfrak{u}_{i} \rightarrow \mathbb{S}$ by

$$
\psi_{i}^{\prime}(x)=\psi_{i}\left(a_{i}+x\right)-\psi_{i}\left(a_{i}\right), \quad x \in \mathfrak{U}_{i} .
$$

The condition (4.1) on $\psi_{i}$ is then equivalent to the assertion that $\psi_{i}{ }^{\prime}$ is a homomorphism of $\mathfrak{U}_{i}$ into $\hat{\mathcal{Y}}$, and $\psi_{i}{ }^{\prime}$ is continuous along with $\psi_{i}$. We may also consider the dual homomorhism $\rho_{i}: \mathbb{B} \rightarrow \hat{\mathfrak{U}}_{i}=\mathfrak{S}_{\mathfrak{V}} / \mathfrak{S}_{i}$, defined $d y$

$$
\left(\psi_{i}{ }^{\prime}(x), g\right)=\left(x, \beta_{i}(g)\right), \quad x \in \mathfrak{U}_{i}=\left(\mathfrak{S}_{\mathbb{E}} / \mathfrak{S}_{i}\right)^{\wedge}, g \in \mathfrak{B} .
$$

In view of the Cohen theorem, the homomorphism $\psi$ is determined by the sets $\Re_{i}, \widetilde{S}_{j}$ and the functions $\beta_{i}$. The notation introduced above will be used in the sequel without further comment. We also use the notation $\rho_{*}$ as the mapping of $L^{*}(\mathfrak{S})$ into $L^{*}(\mathbb{S})$ which is defined by $\rho_{*} f(x)=\rho(x)(f), x \in L(\mathbb{S}), f \in L^{*}(\mathfrak{F})$, whenever $\rho$ is a bounded linear map of $L(\mathfrak{S})$ into $L^{* *}(\mathfrak{S})$.

4.2 Lemma. Let $\lambda$ be a nonsingular homomorphism of 15 into a locally compact abelian group $\Omega$. Then $\lambda$ induces a homomorphism $\rho$ of $L(\mathbb{S})$ into $L^{* *}(\Re)$ such that for $f \in \hat{\Re}, \rho_{*}(f)=f \circ \lambda$.

Proof. For $k \in L^{*}(\Re)$, define $\lambda_{*}(k)$ by

$$
\lambda_{*}(k)(\alpha)=k \circ \lambda(\alpha),
$$

$\alpha \in G$.

We first must show that $\lambda_{*}$ is a well-defined bounded linear mapping of $L^{*}(\Re)$ into $L^{*}(\mathbb{S})$. Suppose that $K_{1}$ and $K_{2}$ are two bounded Borel measurable functions on $\Re$ such that $k_{1}(\beta)=k_{2}(\beta)$ for almost all $\beta$ in $\Re$. Let $\mathbb{F}=\left\{\alpha \in \mathbb{S} \mid k_{1}(\lambda(\alpha)) \neq k_{2}(\lambda(\alpha))\right\}$. Then $\mathbb{F}=\lambda^{-1}(\lambda(\mathbb{F}))$ and by the hypothesis 
of non-singularity $(5$ has measure zero in 8 . Since it is now immediate that $\left|\lambda_{*}(k)(\alpha)\right| \leqq\|k\|$ for almost all $\alpha$ in $\mathscr{S}$, it follows that $\lambda_{*}$ is a bounded linear map of $L^{*}(\Re)$ into $L^{*}(\mathbb{S})$.

For $x \in L(\mathbb{S})$, define $\rho(x)$ on $L^{*}(\Re)$ by

$$
\rho(x)(f)=\lambda_{*} f(x), \quad f \in L^{*}(\Re) .
$$

Clearly $\rho(x) \in L^{* *}(\Re)$, and $\rho$ is a bounded linear mapping from $L(\mathbb{S})$ into $L^{* *}(\Re)$, and $\rho_{*} f=f \circ \lambda$.

We next show that $\rho$ satisfies the multiplicative condition for a homomorphism. Let $x, y \in L(\mathbb{S})$ and $f \in L^{*}(\Re)$. Then

$$
\begin{aligned}
\rho(x y)(f)=\lambda_{*} f(x y) & =\int_{\mathscr{S}} \lambda_{*} f(\alpha) \int_{\mathscr{S}} x(\beta) y(\alpha-\beta) d \beta d \alpha \\
& \left.=\int_{\mathscr{S}} \int_{\mathscr{S}} f(\lambda)(\alpha)\right) x(\beta) y(\alpha-\beta) d \beta d \alpha \\
& =\int_{\mathscr{S}} \int_{\mathscr{S}} f(\lambda(\alpha+\beta)) x(\beta) y(\alpha) d \beta d \alpha .
\end{aligned}
$$

For any $z \in L(\Re)$, and $\delta \in \Re$, it is easily seen [3] that $\langle f, z\rangle(\delta)=$ $\int_{\Omega} f(z+\delta) z(\gamma) d \gamma$. Therefore,

$$
\begin{aligned}
{[\rho(y), f](z) } & =\rho(y)(\langle f, z\rangle)=\lambda_{*}\langle f, z\rangle(y)=\int_{\mathscr{G}} \lambda_{*}\langle f, z\rangle(\alpha) y(\alpha) d \alpha \\
& =\int_{\mathscr{S}}\langle f, z\rangle(\lambda(\alpha)) y(\alpha) d \alpha=\int_{\mathscr{S}} \int_{\mathscr{\Re}} f(\gamma+\lambda(\alpha)) z(\gamma) y(\alpha) d \gamma d \alpha .
\end{aligned}
$$

Since the order of integration may be reversed, we see that for $\gamma \in \mathscr{R}$, $[\rho(y), f](\gamma)=\int_{\mathscr{S}} f(\gamma+\lambda(\beta)) y(\beta) d \beta$. Hence,

$$
\begin{array}{r}
\rho(x) \rho(y)(f)=\rho(x)([\rho(y), f])=\lambda_{*}[\rho(y), f](x)=\int_{\mathscr{G}} \lambda_{*}[\rho(y), f](\alpha) x(\alpha) d \alpha \\
=\int_{\mathscr{S}}[\rho(y), f](\lambda(\alpha)) x(\alpha) d \alpha=\int_{\mathscr{S}} \int_{\mathscr{G}} f(\lambda(\alpha)+\lambda(\beta)) y(\beta) x(\alpha) d \beta d \alpha .
\end{array}
$$

Since we thus have $\rho(x y)(f)=\rho(x) \rho(y)(f)$, for all $f \in L^{*}(K), \rho$ is a homomorphism.

4.3 THEOREM. Let $\mathbb{S}$ and $\mathfrak{S}$ be locally compact abelian groups, with $\mathfrak{S}$ compact. Let $\varphi$ be a homomorphism of $L(\mathbb{S})$ into $M(\mathfrak{C})$. Let $M(\mathfrak{S})$ be regarded as $\left.L^{* *}(\mathfrak{S}) / \mathfrak{Y}\right)^{\perp}(\mathfrak{S})$, and let $\theta$ be the natural mapping of $L^{* *}(\mathfrak{S})$ onto $L^{* *}(\mathfrak{S}) / \mathfrak{Y} \perp(\mathfrak{S})$. Then if each homomorphism $\beta_{i}$, determined by $\varphi$, is nonsingular, there is a homomorphism $\rho$ of $L(\mathbb{B})$ into $L^{* *}(\mathfrak{g})$ such that $\phi=\theta \circ \rho$.

Proof. The justification for considering $M(\mathfrak{2})$ as $L^{* *}(\mathfrak{2}) / \mathfrak{I}^{\perp}(\mathfrak{S})$ is 
Theorem 3,18 of [3].

If $\phi_{*}(f)=0$ for all $f \in \mathfrak{S}_{j}$, define $\rho_{j}: L(\mathbb{S}) \rightarrow L^{* *}(\mathfrak{g})$ by $\rho_{j}(x)=$ $0, x \in L(\mathbb{S})$.

Suppose that $\widetilde{S}_{j} \subset \mathfrak{R}_{i} \subset \widehat{\mathfrak{S}}$, and $\phi_{*}(f)=\psi_{i}(f)$ for $f \in \mathscr{S}_{j}$. In view of (4.1), the homomorphism $\psi_{i}^{\prime}$ of $U_{i}$ into $\widehat{G}$ may be defined by $\psi_{i}{ }^{\prime}(k)=$ $\psi_{i}\left(k+k_{i}\right)-\psi_{i}\left(k_{i}\right)$ for an arbitrary $k_{i} \in \mathfrak{S}_{j}$. The dual homomorphism $\beta_{i}$ of $\mathbb{S}$ into $\mathfrak{E} / \mathfrak{E}_{i}$ is by hypothesis nonsingular. Thus by Lemma 4.2 , there is a homomorphism $\rho_{j}{ }^{\prime}$ of $L(G)$ into $L^{* *}\left(\mathfrak{S}_{2} / \mathfrak{C}_{i}\right)$ such that $\rho_{j *}{ }^{\prime}(k)=k \circ \beta_{i}$, for $k \in\left(\mathfrak{S}_{\mathfrak{C}} / \mathfrak{S}_{i}\right)^{\wedge}=\mathfrak{U}_{i}$.

For $f \in L\left(\mathfrak{S}_{\mathcal{C}} / \mathfrak{S}_{i}\right)$ define $\theta_{i}(f)$ on $\mathfrak{S}$ by $\theta_{i}(f)(\beta)=f\left(\beta+\mathfrak{S}_{i}\right)$. Suppose that the Haar measure on $\mathfrak{S}_{i}$ is normalized so that the measure of $\mathfrak{S}_{i}$ is one. The formula relating integration on a group with that on a quotient group shows that $\theta_{i}$ is an isometric isomorphism of $L\left(\$ / S_{i}\right)$ into $L(\mathfrak{S})$. Thus by Theorem 6.1 of [3], $\theta_{i}{ }^{* *}$ is a homomorphism of $L^{* *}\left(\mathfrak{g} / \mathfrak{S}_{2}\right)$ into $L^{* *}(\mathfrak{g})$. Also for any $u \in L\left(\mathfrak{E} / \mathfrak{S}_{i}\right)$, and $f \in L^{*}(\mathfrak{S})$,

$$
\begin{aligned}
\theta_{i}{ }^{*} f(u) & =f\left(\theta_{i} u\right)=\int_{\mathfrak{S}} f(\beta) \theta_{i}(u)(\beta) d \beta \\
& =\int_{\mathscr{F}_{1} / \mathfrak{F}_{i}} \int_{\mathfrak{S}_{i}} f(\beta+\gamma) \theta_{i}(u)(\beta+\gamma) d \gamma d \dot{\beta},
\end{aligned}
$$

where $d \dot{\beta}$ is the Haar measure on $\mathfrak{S}_{\mathbb{2}} / \mathfrak{L}_{i}$. Thus

$$
\theta_{i}{ }^{*} f(u)=\int_{\mathfrak{F}_{1} / \mathfrak{F}_{i}} u(\dot{\beta}) \int_{\mathfrak{E}_{i}} f(\beta+\gamma) d \gamma d \dot{\beta},
$$

and we conclude that $\theta_{i}{ }^{*} f(\dot{\beta})=\int_{\mathfrak{g}_{i}} f(\beta+\gamma) d \gamma$.

It is well known that in a group algebra the pointwise multiplication by a character is an automorphism of the algebra. We next show that the same situation prevails in the second conjugate algebra of a group algebra. Let $\mathfrak{I}$ be a locally compact abelian group and define, for $\eta \in \hat{\mathfrak{T}}, \eta \circ g$ and $\eta \circ g$ by pointwise multiplication on $\mathfrak{I}$ if $x \in L(\mathfrak{T})$ and $g \in L^{*}(\mathfrak{T})$. Define $\eta \circ G(g)=G(\eta \circ g)$ for $G \in L^{* *}(\mathfrak{T})$. Clearly the map $G \rightarrow \eta \circ G$ is a one-to-one bounded linear map of $L^{* *}(\mathfrak{T})$ onto itself. Let $F, G \in L^{* *}(\mathfrak{I})$ and $g \in L^{*}(\mathfrak{T})$. It remains for us to show that $(\eta \circ F)(\eta \circ G)(g)=\eta \circ(F G)(g)$. Since $(\eta \circ F)(\eta \circ G)(g)=\eta \circ F([\eta \circ G, g])=$ $F(\eta \circ[\eta \circ G, g])$, while $\eta \circ(F G)(g)=F G(\eta \circ g)=F([G, \eta \circ g])$, it suffices if we show that for all $x \in L(\mathfrak{T}), \eta \circ[\eta \circ G, g](x)=[G, \eta \circ g](x)$. Now $\eta \circ[\eta \circ G, g](x)=$ $[\eta \circ G, g](\eta \circ x)=\eta \circ G(\langle g, \eta \circ x\rangle)=G(\eta \circ\langle g, \eta \circ x\rangle)$, while $[G, \eta \circ g](x)=G(\langle\eta \circ g, x\rangle)$, so it suffices if we show that for all $y \in L(\mathfrak{T}), \eta \circ\langle g, \eta \circ x\rangle(y)=\langle\eta \circ g, x\rangle(y)$. Since $\eta \circ\langle g, \eta \circ x\rangle(y)=g((\eta \circ x)(\eta \circ y))=g(\eta \circ x y)=\eta \circ g(x y)=\langle\eta \circ g, x\rangle(y)$, the original assertion follows.

Define the mapping $\rho_{j}$ by

$$
\rho_{j}(x)=k_{i}^{-1} \circ \theta_{i}^{* *} \rho_{j}^{\prime}\left(\psi_{i}\left(k_{i}\right) \circ x\right), \quad x \in L(\mathbb{S}),
$$


where the dot at each occurrence indicates multiplication of the appropriate functions. Since $k_{i} \in \hat{\mathfrak{S}}$, and $\psi_{i}\left(k_{i}\right) \in \widehat{\hat{S}}, \rho_{j}$ is a composite of four homomorphisms and is thus a homomorphism of $L(\mathfrak{S})$ and $L^{* *}(\mathfrak{E})$.

Suppose that $f \in \mathscr{S}_{j} \subset \mathfrak{R}_{i}$, so that $\phi_{*} f=\psi_{i} f$. Since $\Re_{i}$ is a coset of $\mathfrak{U}_{i}$, there is a $k \in \mathfrak{U}_{i}$ such that $f=k_{i}+k$. We use the same notation for $k$ when it is viewed as a member of $\left(\mathfrak{E} / \mathfrak{S}_{i}\right)^{\wedge}$. For any $x \in L(\mathbb{S})$, $\rho_{j *} f(x)=\rho_{j}(x)(f)=k_{i}^{-1} \circ \theta_{i}{ }^{* *} \rho_{j}^{\prime}\left(\psi_{i}\left(k_{i}\right) \circ x\right)(f)=\theta_{i}{ }^{* *} \rho_{j}^{\prime}\left(\psi_{i}\left(k_{i}\right) \circ x\right)(k)=$ $\rho_{j}^{\prime}\left(\psi_{i}\left(k_{i}\right) \circ x\right) \theta_{i}^{*}(k)$. From the formula obtained earlier for $\theta_{i}{ }^{*}$, it is immediate that $\theta_{i}{ }^{*}$ simply transfers $k$ from being viewed as a member of $\mathfrak{U}_{i} \subset \hat{\mathfrak{K}}$, to being viewed as a member of $\left(\mathfrak{S} / \mathfrak{S}_{i}\right)^{\wedge} \subset L^{*}\left(\mathfrak{S} / \mathfrak{S}_{i}\right)$. Thus

$$
\begin{aligned}
\rho_{j *} f(x) & =\rho_{j}^{\prime}\left(\psi_{i}\left(k_{i}\right) \circ x\right)(k)=\int_{\mathscr{G}} \rho_{j}^{\prime}(k)(\alpha) \psi_{i}\left(k_{i}\right)(\alpha) x(\alpha) d \alpha \\
& =\int_{\mathscr{S}}\left(k, \beta_{i}(\alpha)\right) \psi_{i}\left(k_{i}\right)(\alpha) x(\alpha) d \alpha=\int_{\mathscr{S}}\left(\psi_{i}^{\prime}(k), \alpha\right) \psi_{i}\left(k_{i}\right)(\alpha) x(\alpha) d \alpha,
\end{aligned}
$$

by use of (4.3). Thus by use of the definition of $\psi_{i}^{\prime}$ in terms of $k_{i}$, we have

$$
\begin{aligned}
\rho_{j^{*}} f(x) & =\int_{\mathscr{S}}\left(\psi_{i}\left(k+k_{i}\right)-\psi_{i}\left(k_{i}\right), \alpha\right)\left(\psi_{i}\left(k_{i}\right), \alpha\right) x(\alpha) d \alpha \\
& =\int_{\mathscr{S}}\left(\psi_{i}(f), \alpha\right) x(\alpha) d \alpha=\int_{\mathscr{S}} \Phi_{*} f(\alpha) x(\alpha) d \alpha .
\end{aligned}
$$

We therefore conclude that $\rho_{j^{*}} f(x)=\varphi_{*} f(x)$ for all $x \in L(\mathbb{B})$ or that $\rho_{i^{*}} f=\phi_{*} f$ for $f \in \widetilde{S}_{i}$.

Now, by the Cohen theorem, $\hat{\mathfrak{F}}$ is the disjoint union of the sets $\mathfrak{S}_{j}$. The characteristic function of $\mathfrak{S}_{j}$ is then the Fourier transform of an idempotent measure in $M(\mathfrak{S})=L^{* *}(\mathfrak{S}) / \mathfrak{Y} \perp(\mathfrak{S})$. Let $F_{j}$ be any member of $L^{* *}(\mathfrak{g})$ such that $\theta F_{j}$ is the Fourier transform of the characteristic function of $\subseteq_{j}$. Then $F_{j}^{2}-F_{j} \in \mathfrak{Y} \perp(\mathfrak{C})$. Now, Theorem 3.15 of [3] states that $\mathfrak{Y}^{\perp}(\mathfrak{S})$ is the radical of $L^{* *}(\mathfrak{S})$, and therefore Theorem 2.3.9 of [5] yields $E_{j} \in L^{* *}(\mathfrak{S})$ such that $E_{j}^{2}=E_{j}$ and $\theta E_{j}=\theta F_{j}$.

We next show that if $i \neq j$, then $E_{i} F E_{j}=0$ for any $F \in L^{* *}(\mathfrak{g})$. suppose that $f \in \widehat{\mathfrak{S}}$, then Lemma 3.6 of [3] yields

$$
E_{i} F E_{j}(f)=E_{i}(f) F(f) E_{j}(f) .
$$

For $f \in \hat{\mathfrak{Y}}, E_{k}(f)=F_{k}(f)=\chi\left(\Im_{k}\right)(f)$, where $\chi\left(\Im_{k}\right)$ is the characteristic function of $S_{k}$. Thus since $S_{i}$ and $S_{j}$ are disjoint $E_{i} F E_{j}(f)=0$. Hence $E_{i} F E_{j} \in \mathfrak{Y}^{\perp}$, the radical of $L^{* *}(\mathfrak{g})$. For a compact group $\mathfrak{S}$, the radical is also the right annihilator of $L^{* *}(\mathfrak{2})$ by Theorem 3.5 of [3]. Thus since $E_{i}=E_{i}^{2}, E_{i} F E_{j}=E_{i}\left(E_{i} F E_{j}\right)=0$.

Let $\rho$ be defined on $L(\mathbb{S})$ by

$$
\rho(x)=E_{1} \rho_{1}(x) E_{1}+\cdots+E_{r} \rho_{r}(x) E_{r}, \quad x \in L(\mathbb{S}),
$$

where $\widehat{\hat{\mathfrak{S}}}=\mathfrak{S}_{1} \cup \cdots \cup \mathfrak{S}_{r}$. Clearly $\rho$ is a bounded linear transformation of $L(\mathbb{S})$ into $L^{* *}(\mathfrak{S})$, and to see that $\rho$ is a homomorphism it suffices if 
we show that $E_{i} \rho_{i}(x y) E_{i}=E_{i} \rho_{i}(x) E_{i} \rho_{i}(y) E_{i}$. The latter equality is ertablished by an identical argument to that used above to show $E_{i} F E_{j}=$ 0 for $i \neq j$. Thus $\rho$ is a homomorphism of $L(\mathbb{S})$ into $L^{* *}(\mathfrak{S})$.

To see that $\theta \circ \rho=\varphi$, it suffices if we show that $\varphi_{*}(f)=$ $(\theta \circ \rho)_{*}(f)$ for $f \in \hat{\mathfrak{S}}$. Suppose that $f \in \mathfrak{S}_{k}$. Then for $x \in L(\mathbb{S}),(\theta \circ \rho)_{*}(f)(x)=$ $\theta \circ \rho(x)(f)=E_{k} \rho_{k}(x) E_{k}(f)$, since $E_{i}(f)=0$ if $i \neq k$. Thus $(\theta \circ \rho)_{*}(f)(x)=$ $\rho_{k}(x)(f)=\varphi_{*} f$ as was shown earlier.

\section{REFERENCES}

1. Richard Arens, Operations in function classes, Monat. für Math. 55 (1951), 1-19.

2. - The adjoint of a bilinear operation, Proc. Amer. Math. Soc., 2 (1954), 839-848. 3. P. Civin and B. Yood, The second conjugate space of a Banach algebra as an algebra, Pacific J. Math., 11 (1961), 820-847.

4. P. J. Cohen, On homomorphisms of group algebras, Amer. J. Math., 82 (1960), 213-226.

5. C. E. Rickart, General Theory of Banach Algebras, Van Nostrand, New York, 1960.

6. André Weil, $L$ ' intégration dans les groupes topologique et ses appicationes, Herman et Cie, Paris, 1953.

UNIVERSITY OF FLORIDA

UNIVERSITY OF OREGON 
